\title{
Polar nanoregions and single crystal diffuse scattering in relaxor ferroelectrics
}

Richard Welberry, Research School of Chemistry, Australian National University, Canberra, ACT 2614, Australia

Relaxor ferroelectrics have attracted tremendous attention in recent years because of their extraordinary dielectric and piezoelectric properties. Of these materials the lead-based perovskite relaxors, $\mathrm{Pb}\left(\mathrm{Zn}_{1 / 3} \mathrm{Nb}_{2 / 3}\right) \mathrm{O}_{3}(\mathrm{PZN})$ and $\mathrm{Pb}\left(\mathrm{Mg}_{1 / 3} \mathrm{Nb}_{2 / 3}\right) \mathrm{O}_{3}$ ( $\left.\mathrm{PMN}\right)$ have been two of the most extensively studied. Despite this, the exact nature of the structure and how it relates to its relaxor properties is still the subject of much controversy. It is now widely believed that fundamental to an understanding of their unique properties is the existence of polar clusters (or polar nanoregions - PNRs). The nature of PNRs - their morphology and dynamics - has been the subject of intense debate in recent years but so far this has not yielded a definitive nanoscale picture of relaxors.

Diffuse scattering (DS) stands out as a method that can provide direct access to local structural information in a material. It enables extraction of details concerning the correlations between atoms over the length-scale of a few nanometers. Neutron DS is particularly important for studying relaxors as unlike $\mathrm{x}$-rays it offers a cross-section for oxygen comparable to those for the metal ions. However, as is the case for all diffraction experiments, because the intensity is the Fourier transform of the pairdistribution function only, its interpretation is subject to ambiguity.

In this paper we review the development of models describing the PNRs from early qualitative models through to atom-based models built using density functional theory (DFT)-derived potentials.

(a) PNRs in a simple qualitative model

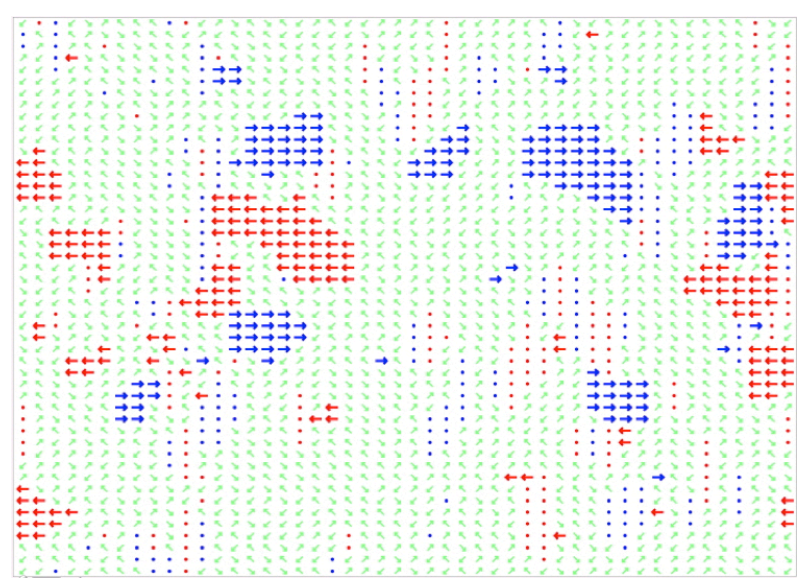

(b) MD simulation results in an atom-based model

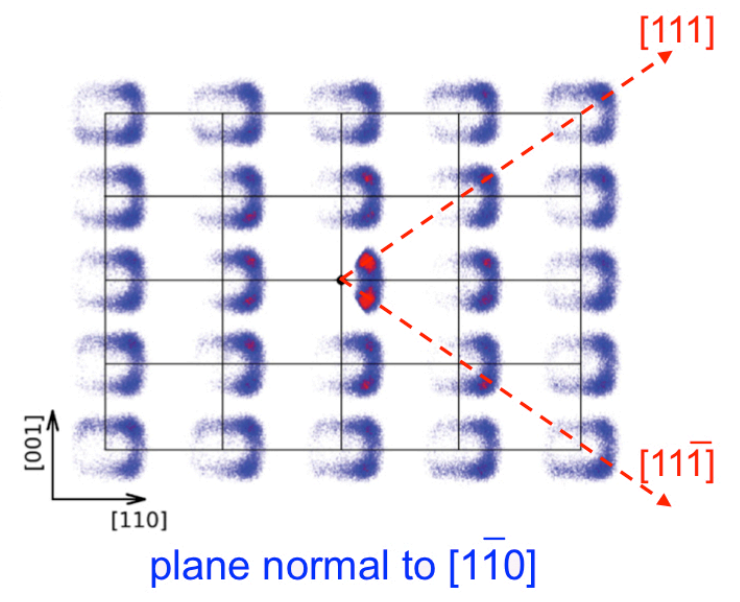

